\title{
Impedance-Based Synchronization of Active Rectifier in Inductive Power Transfer Systems
}

\author{
Guodong $\mathrm{Zhu}^{1}$ and Dawei Gao ${ }^{2}$ \\ ${ }^{1,2}$ State Key Laboratory of Automotive Safety and Energy, School of Vehicle and Mobility, \\ Tsinghua University, Beijing 100084, China \\ dwgao@mail.tsinghua.edu.cn
}

\begin{abstract}
Active rectification is a common option for improving efficiency and impedance matching in inductive power transfer systems. One of the technical challenges in active rectification is the synchronization of phase angle. In this work, the rectifier input impedance, which is calculated from the AC current and $\mathrm{AC}$ voltage, is used as the control objective during phase synchronization. When the impedance angle matches the target value, synchronization of phase is automatically fulfilled. The details of a PI-controller-based phase synchronization algorithm is introduced, and the PI coefficients are manually optimized. Experimental results demonstrate the good performance of the proposed phase synchronization method.
\end{abstract}

Keywords: AC impedance, active rectifier, inductive power transfer, phase synchronization.

\section{$1 \quad$ Introduction}

Recent research on inductive power transfer (IPT) is increasingly focused on improving the system performance, after the basic operating principles and optimization techniques have been thoroughly studied. Among the concerns in system design, energy efficiency and system controllability remain important ones. On the receiver (RX) side, active rectifiers are commonly adopted to reduce the forward voltage drop on the power switches and increase the degree of freedom. For instance, the two-stage load regulation scheme consisting of a passive rectifier and a DC/DC converter [1][2] can be replaced by a single-stage scheme which contains an active rectifier only [3]-[6].

Active rectification is commonly applied in maximum efficiency tracking [5], [7] and bi-directional power transfer [3]-[4], [8]. In the former case, active rectifiers are responsible for matching the load impedance to the optimal value to maximize the system efficiency. In the latter case, active rectifiers enable the power flow direction to be reversed. Active rectification has its own challenges in that extra PWM driving signals are needed. Because the inverter and the rectifier are driven by separate MCUs, phase synchronization is indispensable. 
Zero-crossings $(\mathrm{ZCs})$ of the rectifier input current $\left(\mathrm{i}_{\text {rec }}\right)$ are usually employed as the hardware synchronization signal of the PWM module [5]-[6], [8]-[10]. Besides, phase-locked loop (PLL) is adopted to regulate the phase difference between $\mathrm{i}_{\text {rec }}$ and the PWM signals. Despite having a simple hardware structure, this scheme is susceptible to noises, as is analyzed in [11] and proved by an example therein. The example reveals that the PWM signal may fail to toggle when the $\mathrm{ZC}$ of $\mathrm{i}_{\text {rec }}$ is advanced due to frequency dithering. In some references, an extra phase-sensing coil is adopted on the RX side to acquire the phase of the primary coil current [4]-[5]. The induced voltage in the extra coil and $\mathrm{i}_{\text {rec }}$ are combined to yield the phase difference between the coil currents. Obviously, the extra coil increases system complexity. All these schemes are based on hardware synchronization signals that are directly connected to the PWM module and synchronization is executed cycle-by-cycle.

The scheme proposed in [11] combines the PLL method with the proposed "chained trigger" method, i.e., multiple signals are arranged in a specific order. From front to end, the signals are: $\mathrm{i}_{\mathrm{rec}}, \mathrm{PWM} 1, \mathrm{PWM} 2$ and PWM3. They are connected in such a way that the phase of each signal (except $\mathrm{i}_{\mathrm{rec}}$ ) is synchronized when the one preceding it encounters a ZC. The rectifier driving signals are based on PWM2 and PWM3 and almost immune to the dithering in the $\mathrm{ZC}$ of $\mathrm{i}_{\text {rec }}$ due to one restriction: the instant at which PWM2 toggles is kept away from the $\mathrm{ZC}$ of $\mathrm{i}_{\text {rec }}$ by a certain margin. However, the restriction means that the phase of PWM signals cannot be completely freely regulated. Besides, the hardware circuitry is relatively complex.

There are two deficiencies common to cycle-by-cycle hardware-triggered synchronization methods: (1) the phase accuracy of the PWM signals is affected by the noises in the $\mathrm{ZC}$ of $\mathrm{i}_{\text {rec }}$ and such an error cannot be eliminated because synchronization is executed every switching cycle and (2) without $\angle Z_{\text {rec }}$ feedback, the actual phase difference between the rectifier input voltage and $\mathrm{i}_{\mathrm{rec}}$ cannot be accurately controlled due to adverse factors such as dead bands.

To avoid the shortcomings associated with cycle-by-cycle hardware-triggered direct synchronization, this work aims to develop a simple indirect synchronization method based on regulating the rectifier input impedance angle $\left(\angle Z_{\text {rec }}\right)$. The main technical features are as follows: (1) Phase synchronization is based on the direct control of $\angle Z_{\text {rec }}$ to guarantee the accuracy of $\angle Z_{\text {rec }}$. (2) Although the ZCs of $i_{\text {rec }}$ affect the measured $\angle Z_{r e c}$, they are not directly connected to the synchronization pin to reset the CTR registers, hence noises in the ZCs can be effectively filtered. (3) The PWM control registers are changed in the PI-controller-based phase synchronization algorithm (PSA), which is executed at a relatively low frequency (designated as $f_{p s}$ ), instead of cycle-by-cycle. Between two neighboring PSA executions, the PWM control signals are unchanged and thus immune to high-frequency noises.

The rest of this paper is arranged as follows. Section 2 introduces the mathematical model of the IPT system. Section 3 analyzes the active rectifier control strategy from two aspects: the operating principle of PSA and the "tooth missing" phenomenon. Section 4 demonstrates the experimental results under both static and dynamic conditions. Section 5 gives the conclusions. 


\section{Mathematical Model of IPT System}

\subsection{Circuit Model}

As is a common practice, only the fundamental current and voltage components are considered while the harmonics are omitted when analyzing the power transfer performance of the IPT system. With the coils compensated using the LCC topology, the simplified circuit diagram of an IPT system is shown in Fig. 1. The transmitter (TX) and receiver $(\mathrm{RX})$ coils $\left(L_{1}\right.$ and $\left.L_{2}\right)$ are coupled by the mutual inductance M. The LCC compensation networks are composed of $L_{f 1}, L_{f 2}, C_{1}, C_{2}, C_{f 1}$ and $C_{f 2}$. $\boldsymbol{U}_{\text {inv }}$ is the inverter output voltage and $\boldsymbol{U}_{\text {rec }}$ is the rectifier input voltage. The input impedance of the rectifier is designated as $Z_{r e c}$. In the following analysis, $f_{i n v}$ and $f_{\text {rec }}$ refer to the switching frequency of the inverter and the rectifier, respectively. According to the superimposition principle, $\boldsymbol{I}_{\text {rec }}$ contains two components, one contributed by $\boldsymbol{U}_{\text {inv }}$ and the other by $\boldsymbol{U}_{\text {rec }}$ :

$$
\boldsymbol{I}_{\text {rec }}=\boldsymbol{I}_{\text {rec1 } 1}+\boldsymbol{I}_{\text {rec } 2}=\alpha \boldsymbol{U}_{\text {inv }}+\beta \boldsymbol{U}_{\text {rec }}
$$

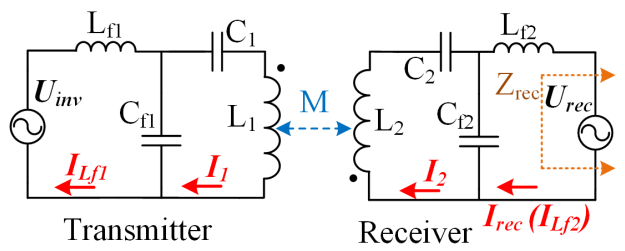

Fig. 1. Simplified circuit diagram of an LCC-compensated IPT system.

where the coefficients $\alpha$ and $\beta$ are related to $f_{\text {inv }}$ and $f_{\text {rec }}$ as well as the circuit parameters. $\boldsymbol{I}_{\boldsymbol{r e c} \mathbf{1}}$ is derived when $\boldsymbol{U}_{\boldsymbol{r e c}}$ is short-circuited, and $\boldsymbol{I}_{\boldsymbol{r e c} \mathbf{2}}$ is similarly derived. In the time domain, the two components are formulated as

$$
\begin{gathered}
i_{\text {rec } 1}(t)=\left|\boldsymbol{I}_{\text {rec } 1}\right|(t) \cdot \sin \left(\omega_{\text {rec }} t+\Delta \omega t\right) \\
i_{\text {rec } 2}(t)=\left|\boldsymbol{I}_{\text {rec } 2}\right|(t) \cdot \sin \left(\omega_{\text {rec }} t\right)
\end{gathered}
$$

where $\omega_{\text {rec }}=2 \pi f_{\text {rec }}$ and $\Delta \omega=2 \pi\left(f_{\text {inv }}-f_{\text {rec }}\right)$. Replacing the equations into (1) yields

$$
\begin{aligned}
i_{\text {rec }}(t)= & \left|\boldsymbol{I}_{\text {rec } \mathbf{1}}\right|(t) \sin \left(\omega_{\text {rec }} t\right) \cos (\Delta \omega t)+\left|\boldsymbol{I}_{\text {rec } \mathbf{1}}\right|(t) \cos \left(\omega_{\text {rec }} t\right) \sin (\Delta \omega t) \\
& +\left|\boldsymbol{I}_{\text {rec } 2}\right|(t) \sin \left(\omega_{\text {rec }} t\right)
\end{aligned}
$$

Even when $\left|\boldsymbol{I}_{\text {rec } \mathbf{1}}\right|$ and $\left|\boldsymbol{I}_{\text {rec } \mathbf{2}}\right|$ are constant, the amplitude of $\boldsymbol{I}_{\text {rec }}$ is time-varying due to the first and second terms in (3). When $\left|f_{\text {inv }}-f_{\text {rec }}\right|$ is significantly smaller than $f_{\text {inv }}, \cos (\Delta \omega t)$ and $\sin (\Delta \omega t)$ vary very slowly with time, hence $\boldsymbol{I}_{\text {rec }}$ can be simplified as a phasor with a slowly varying amplitude. 


\subsection{Instantaneous Rectifier Input Impedance}

In this work, the instantaneous frequency-domain rectifier input impedance is defined as

$$
Z_{\text {rec }}=\frac{\left|U_{\text {rec }}\right|(t)}{\left|\boldsymbol{I}_{\text {rec }}\right|(t)} \cdot e^{j\left(\omega_{\text {rec }} \Delta t\right)}
$$

where $\Delta t$ is the time difference by which the zero-crossing of $\boldsymbol{U}_{\text {rec }}$ leads that of $\boldsymbol{I}_{\text {rec }}$. $\Delta t$ is affected by $\left|\boldsymbol{I}_{\text {rec } \mathbf{1}}\right|,\left|\boldsymbol{I}_{\text {rec } \mathbf{2}}\right|, \omega_{\text {rec }}$ and $\Delta \omega$. Besides, $\Delta t$ is time-varying when $\left|f_{\text {inv }}-f_{\text {rec }}\right| \neq 0$. Only when $f_{\text {inv }}=f_{\text {rec }}$ and the rectifier driving signals have correct phase angles can $Z_{\text {rec }}$ reach the target value. When $\left|f_{\text {inv }}-f_{\text {rec }}\right| \neq 0$ and no intervention is applied, $Z_{\text {rec }}$ varies slowly with time, which destabilizes the output power.

Because the inverter and the rectifier are driven by separate MCUs, the inequality between $f_{\text {inv }}$ and $f_{\text {rec }}$ is unavoidable even if the same MCU and the same switching frequency are adopted on both sides. The phase of the PWM signals should be adjusted to maintain $\angle Z_{\text {rec }}$ at the target value. In fact, $\left|f_{i n v}-f_{\text {rec }}\right|$ is typically smaller than $f_{\text {inv }}$ by several orders of magnitude, hence the adjustment, i.e., PSA, can be executed at a low frequency.

$Z_{r e c}$ is calculated from the rectifier input current and voltage, the acquisition of which will be introduced in the subsequent section. $Z_{\text {rec }}$ is selected as the control target in this work for two reasons: (1) A stable $\angle Z_{\text {rec }}$ implies that the active rectifier has been successfully synchronized to the inverter, i.e., the $\left|f_{\text {inv }}-f_{\text {rec }}\right|$ error is compensated. Compared to other quantities, $\angle Z_{\text {rec }}$ is easy to measure. (2) It is apparent that $Z_{r e c}$ is an important factor that affects system performance, e.g., power transfer efficiency, hence the ability to maintain it at a suitable value is of practical interest, although searching for the optimal $Z_{r e c}$ is out of the scope of this work.

\section{Active Rectifier Control}

In this work, the rectifier conduction angle $\left(\theta_{\text {rec }}\right)$ and the rectifier phase angle $\left(\alpha_{\text {rec }}\right)$ are the control variables in obtaining the target $Z_{r e c}$. A graphical illustration of these two parameters is given in Fig. 2. The control of $Z_{\text {rec }}$ is simple: when $\left|Z_{\text {rec }}\right|$ is below the target value, $\theta_{\text {rec }}$ should be increased, and vice versa. On the other hand, $\alpha_{\text {rec }}$ should strictly follow the target $\angle Z_{\text {rec }}$ value.

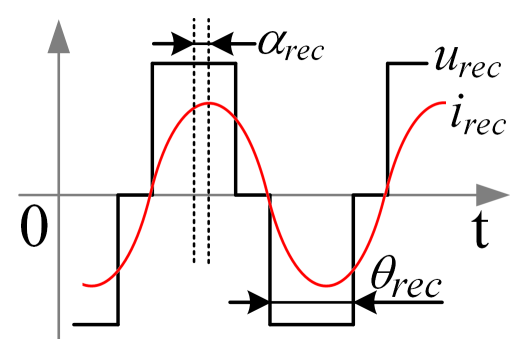

Fig. 2. Definition of $\theta_{r e c}$ and $\alpha_{r e c}$. In this example, $\alpha_{r e c}>0$. 


\subsection{Design of Current and Voltage Sense Circuits}

The amplitude and phase of $\boldsymbol{U}_{\text {rec }}$ and $\boldsymbol{I}_{\text {rec }}$ are acquired using the current and voltage sense circuits shown in Fig. 3, which are simplified versions of the design introduced in [12]. The circuits output a DC voltage signal that corresponds to $\left|\boldsymbol{U}_{\text {rec }}\right|$ or $\left|\boldsymbol{I}_{\text {rec }}\right|$ and a digital signal that contains the phase information. On the MCU side, the ADC and capture modules are required to process the output signals $\left(\boldsymbol{U}_{\text {vol }}\right.$ or $\left.\boldsymbol{U}_{\boldsymbol{c u r}}\right)$. Changes in $\boldsymbol{U}_{\boldsymbol{r e c}}$ or $\boldsymbol{I}_{\boldsymbol{r e c}}$ are immediately detected due to the low time constant of the sense circuits.

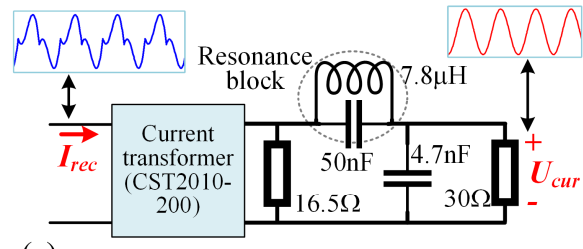

(a)

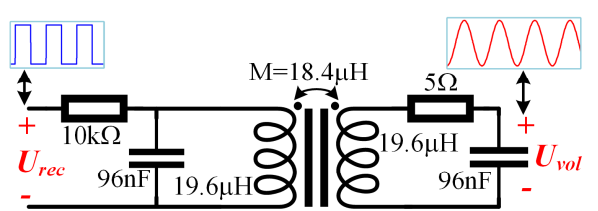

(b)

Fig. 3. Design of the current and voltage sense circuits. (a) Current sense circuit. (b) Voltage sense circuit.

\subsection{Introduction of the PWM Generation Scheme}

TMS320F28335 is adopted in this work. The eCAP module measures $\angle Z_{\text {rec }}$ based on the ZCs of $\boldsymbol{U}_{\text {rec }}$ and $\boldsymbol{I}_{\text {rec }}$ and triggers an interrupt at a low frequency. PSA is executed in the interrupt functions according to the operating principle illustrated in Fig. 4 (a), hence $f_{p s}$ equals the interrupt frequency. The phase of the PWM output signal is regulated by either the CMP register or the PHS register. The "tooth missing" phenomenon in Fig. 4 (b) will be analyzed in Section 3.4.
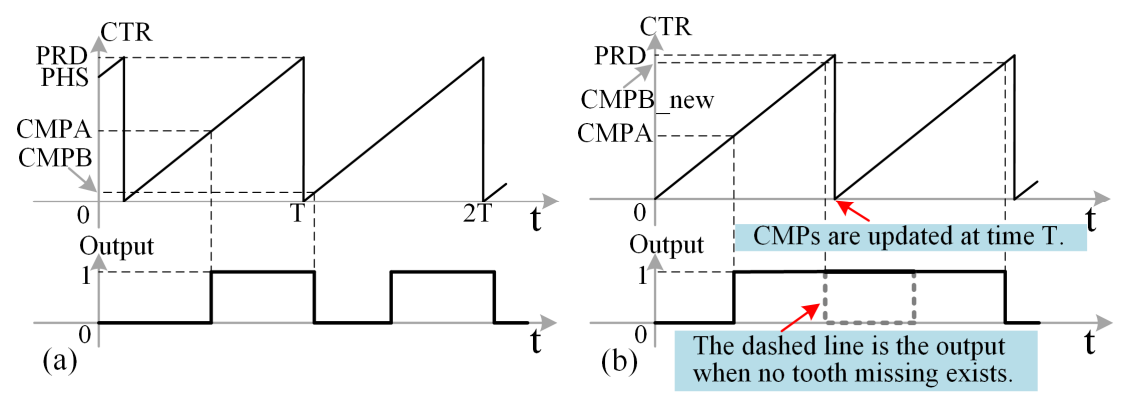

Fig. 4. Operating principle of the PWM module. (a) Normal operation. (b) "Tooth missing" caused by the negative zero-crossing of CMPB.

In the full-bridge active rectifier, both half-bridges are driven by 50\%-duty-ratio PWM signals with a phase difference determined by $\theta_{\text {rec }}$. CMPA and CMPB are updated in accordance with $\Delta \angle Z_{\text {rec }}$. When $\angle Z_{\text {rec }}$ exceeds the target value, the CMPs are increased so that the PWM output and the resultant $\boldsymbol{U}_{\text {rec }}$ are delayed, and vice 
versa. The CMP registers of the two half-bridges are the same, and $\theta_{\text {rec }}$ is adjusted via the phase register of the second half-bridge (PHS2). To sum up, $\theta_{\text {rec }}$ is determined by PHS 2 while $\alpha_{\text {rec }}$ is determined by the CMP registers.

\subsection{Impedance-Angle-Based Phase Synchronization Algorithm}

When no intervention is applied, $\angle Z_{\text {rec }}$ gradually deviates from its target value when there exists a slight difference between $f_{i n v}$ and $f_{\text {rec }}$. In this work, the algorithm illustrated in Fig. 5 is adopted for phase synchronization. Using a simple PI controller, the CMPs are adjusted based on the difference between $\angle Z_{r e c}$ and its target value $\left(\angle Z_{\text {rec,target }}\right)$. The operating principle is as follows. Once $\Delta Z_{\text {rec }}$ exceeds a certain threshold, the CMPs are changed to minimize the difference, i.e., $\Delta Z_{r e c}$. On the other hand, PRD remains constant as it is determined by $f_{\text {rec }}$ only. The CTR registers run freely (unaffected by PSA). Using this approach, not only is the clock frequency error compensated, but the target $\angle Z_{\text {rec }}$ is also reached. Because $\left|f_{\text {rec }}-f_{\text {inv }}\right| \ll f_{\text {inv }}$, $\Delta \angle Z_{\text {rec }}$ accumulates slowly and can be easily compensated.

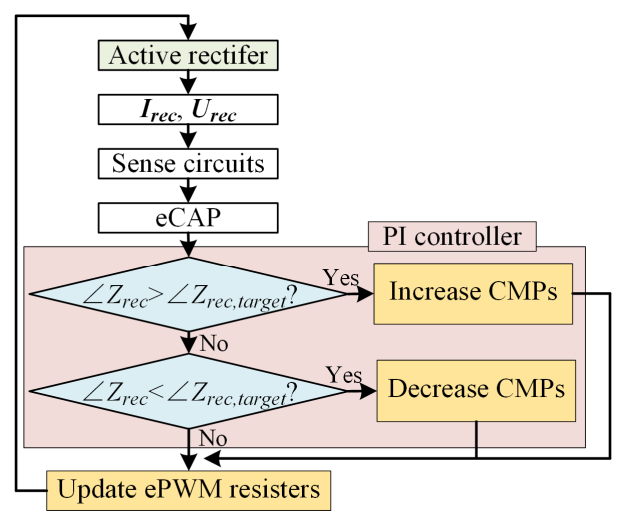

Fig. 5. Phase synchronization algorithm (PSA).

Although the PWM resisters accept integers only, they can be varied continuously during PSA. In this work, PI control is adopted for phase synchronization: the input is $\Delta \angle Z_{\text {rec }}$ and the output is a float-type CMPA increment value (CMPA_INC). When |CMPA_INC| exceeds 1, the increment to CMPA and CMPB becomes effective.

\section{4 "Tooth Missing" Phenomenon}

CMPA and CMPB are between 0 and PRD, hence the zero-crossing of the CMPs is inevitable. In this work, positive zero-crossing refers to the CMPs returning to zero after surpassing PRD and negative zero-crossing is defined as the CMPs rising to a value near PRD after it is decreased to zero. When negative zero-crossings occur, the PWM output may fail to toggle, which is referred to as "tooth missing" in this work. An example is given in Fig. 4 (b). When $C T R$ reaches 0 and $C M P B \approx 0$, the $P W M$ output is just about to toggle. However, because CMPB has been updated to a value 
close to PRD, i.e., CMPB new $\approx$ PRD, the condition for the output to be pulled low is lost and the output remains high over the entire period that follows. An actual waveform is given in Fig. 6 (a). The consequence is severe oscillations in $i_{\text {rec }}$.

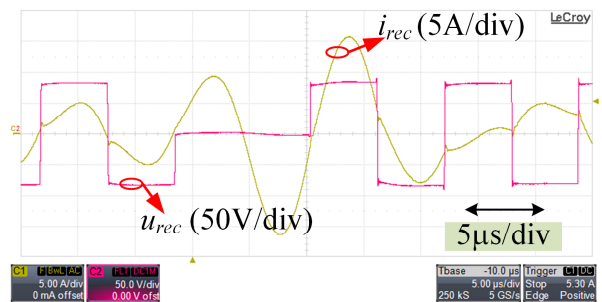

(a)

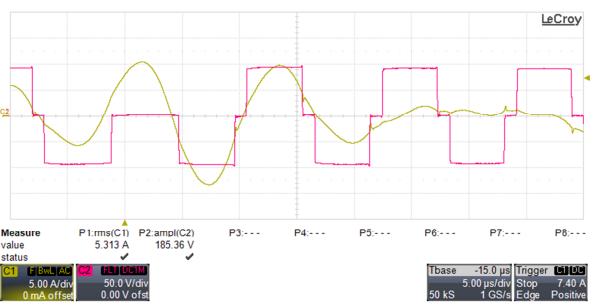

(b)

Fig. 6. The "tooth missing" phenomena. (a) Full-period tooth missing. (b) Half-period tooth missing.

Besides, the increase of PHS2 is the potential cause of another type of tooth missing, an actual waveform of which is given in Fig. 6 (b). One can infer from Fig. 4 that when $\mathrm{CMPA}=\mathrm{PHS} 2$ but PHS2 is increased and updated at $\mathrm{t}=0$, the PWM output may fail to be pulled high because CTR is forced to equal PHS2, which is greater than CMPA, before the condition $\mathrm{CTR}=\mathrm{CMPA}$ occurs. The result is that PWM2 remains low for the entire period that follows. Meanwhile, the other half-bridge is in normal operation, and the $u_{\text {rec }}$ waveform is as shown in Fig. 6 (b). These two types are referred to as full-period and half-period tooth missing events, respectively. Although the oscillations in $i_{\text {rec }}$ will eventually be attenuated, constant occurrence of tooth missing results in acoustic noise, mechanical vibration and degradation of efficiency.

The tooth missing phenomenon is intrinsic to all timer-based PWM generation schemes. With sufficiently low PI coefficients and a small $f_{p s}$, tooth missing rarely occurs and its impact is trivial. The negative effect is slower dynamic response. $\angle Z_{\text {rec }}$ is measured at the instants of eCAP events (CEVT1-CEVT4) only, i.e., $\angle Z_{\text {rec }}$ is measured at a frequency of $4 f_{p s}$, hence another benefit of decreasing $f_{p s}$ is that the majority of noises in $i_{\text {rec }}$, which may cause phase errors in the PWM signals, are unnoticed by the MCU.

\section{$4 \quad$ Experimental Results}

\subsection{EV Wireless Charger Prototype Configuration}

The EV wireless charger prototype is shown in Fig. 7. Cree C3M0075120J is used in the inverter and the rectifier. The DC load resistance is $25 \Omega .\left|\boldsymbol{I}_{\mathbf{1}}\right|$ is fixed and the output power varies with the instantaneous $Z_{\text {rec }}$. The waveforms of $u_{\text {rec }}$ and $i_{\text {rec }}$ are acquired using an oscilloscope (Teledyne LeCroy WaveRunner 625Zi). The power and efficiency data are measured using a power analyzer (HIOKI PW6001). The other data are measured using the on-board sensors. Parameters of the compensation circuits are given in Table 1. 


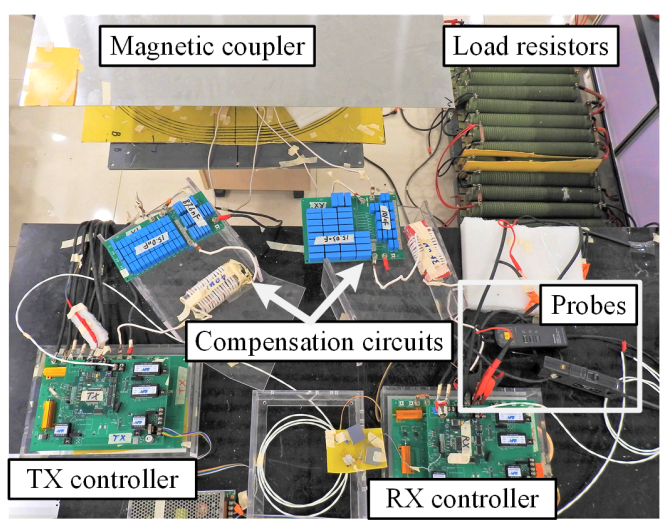

Fig. 7. EV wireless charger prototype.

Table 1. Parameters of the prototype.

\begin{tabular}{cccl}
\hline Parameter & Value $(\mu \mathrm{H})$ & Parameter & Value $(\mathrm{nF})$ \\
\hline$L_{f 1}$ & 31.3 & $C_{f 1}$ & 113 \\
$L_{1}$ & 254.8 & $C_{1}$ & 15.6 \\
$L_{f 2}$ & 31.1 & $C_{f 2}$ & 113 \\
$L_{2}$ & 191.2 & $C_{2}$ & 21.8 \\
$\mathrm{M}$ & 42 & & \\
\hline
\end{tabular}

\subsection{Experimental Results of Static and Dynamic Performance}

Steady-State System Smoothness. The experimental results of static performance are presented in Fig. 8. PSA is executed in the eCAP-triggered interrupts at a $f_{p s}$ of $2.7 \mathrm{kHz}$. When the PI coefficients are small, both $\angle Z_{\text {rec }}$ and $\left|Z_{\text {rec }}\right|$ are very stable. Tooth missing rarely occurs (every few minutes, if there is any). The waveforms are as smooth as those obtained in passive rectification mode. When I or both P and I are increased tenfold, the frequency of tooth missing is significantly increased, as is evidenced by the ripples in $\left|Z_{\text {rec }}\right|$. When $\mathrm{P}$ alone is increased tenfold, the impact on tooth missing is much weaker and the system still operates smoothly. Therefore, to expedite dynamic response, the $\mathrm{P}$ coefficient can be increased to a suitable level while the I coefficient should be much lower. The final choice of $\mathrm{P}$ and $\mathrm{I}$ is 0.18 and 0.02 , respectively. Experimental results demonstrate that when $f_{p s}$ is improved under the restriction that the PI coefficients are inversely proportional to $f_{p s}$, the static performance is almost unaffected. 

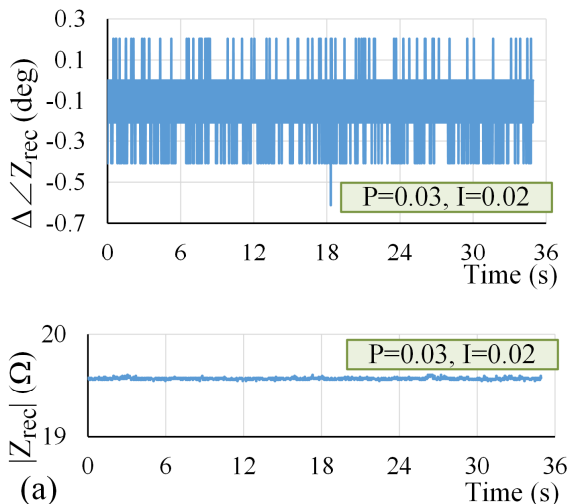
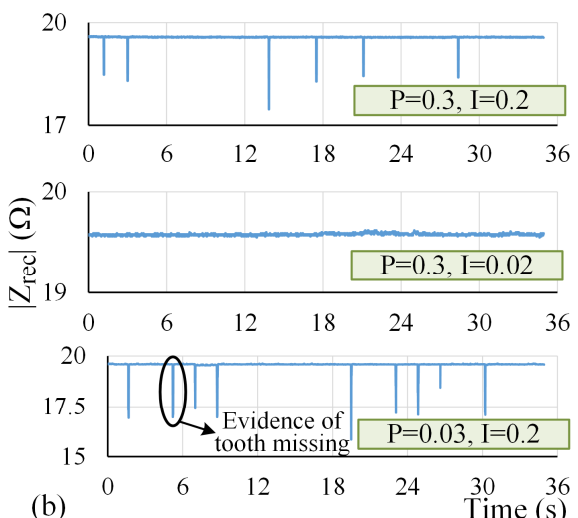

(b) $\begin{array}{llll}0 & 12 & 18 & 24\end{array}$

Fig. 8. Impact of PI coefficients on steady-state system smoothness. The output power is $900 \mathrm{~W}$ and the system efficiency is $94.07 \%$. $\alpha_{r e c}=-10^{\circ}$ and $\theta_{\text {rec }}=180^{\circ}$. (a) $\Delta \angle Z_{\text {rec }}$ and $\left|Z_{\text {rec }}\right|$ under low PI coefficients. (b) $\left|Z_{\text {rec }}\right|$ when the PI coefficients are increased.

Dynamic Performance. Step responses of $\alpha_{r e c}$ and $\theta_{r e c}$ are tested to examine the dynamic performance of the proposed PSA. $\alpha_{r e c}$ and $\theta_{r e c}$ are controlled by CMPs and PHS2, respectively. CMPs are regulated in the eCAP-triggered interrupts while PHS2 is regulated in the timer-triggered interrupts. The frequency of both interrupts is $2.7 \mathrm{kHz}$ and $1.0 \mathrm{kHz}$, respectively, hence the burden on the MCU is light. Both the target $\alpha_{r e c}$ and the target $\theta_{\text {rec }}$ are varied at a gradient of $\pm 4 \% \mathrm{~ms}$.

The test results in Fig. 9 demonstrate the transition times, the frequency of tooth missing and the ability of the system to operate smoothly. Using the coefficients " $\mathrm{P}=0.18, \mathrm{I}=0.02$ ", the MCU has no difficulty in keeping up with the gradient. Approximately $35 \mathrm{~ms}$ and $47 \mathrm{~ms}$ are needed to change $\theta_{\text {rec }}$ and $\alpha_{\text {rec }}$ by 30 degrees, respectively. It is observed during the tests that both the dynamic response speed and the occurrence frequency of tooth missing increase monotonically with the gradients. Therefore, the choice of the gradients depends on whether dynamic response speed is of the highest priority. If system smoothness is an important concern, then moderate gradients should be adopted. The response speed and system smoothness reflected in Fig. 9 is quite acceptable for most static inductive battery chargers as both the mutual inductance and the target charging current vary slowly with time.

The waveforms over a relatively long period of time are presented in Fig. 9. By contrast, the details during the tooth-missing events are not shown as they are related to the circuit dynamics of the IPT system, which is not the focus of this work. 

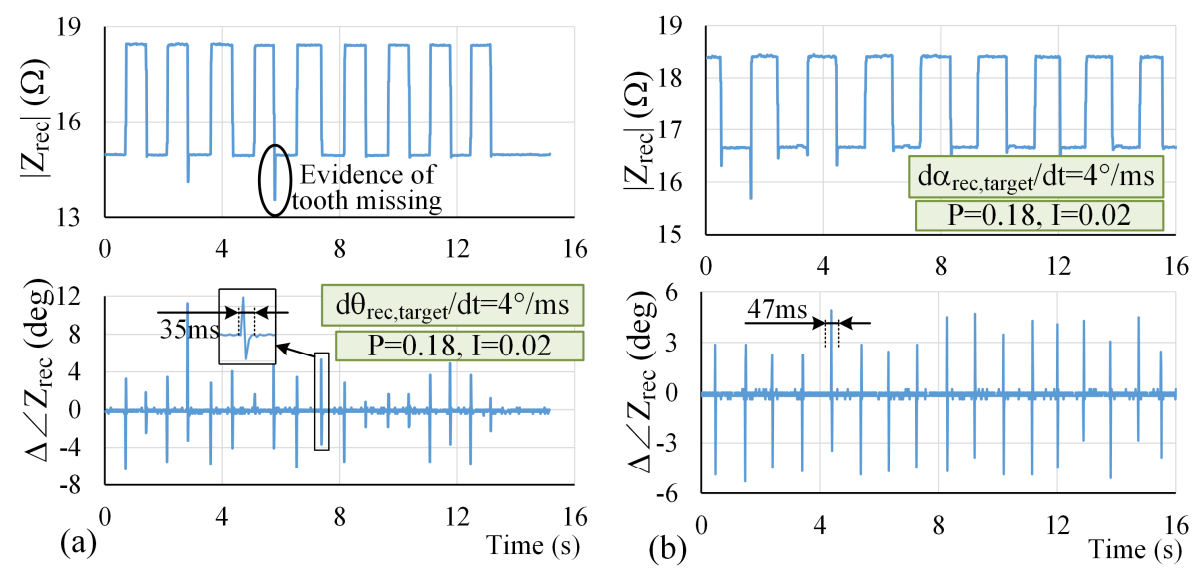

Fig. 9. Dynamic performance. (a) Step response of $\theta_{\text {rec }}$ between 120 and 150 degrees. (b) Step response of $\alpha_{\text {rec }}$ between 0 and -30 degrees.

\subsection{Discussions}

In this work, the choice of PI coefficients is mainly based on experience as the relevant technical know-how is well-established. Both the static and dynamic performance of the proposed phase synchronization method is good. PSA is executed at a relatively low frequency, hence the burden on the MCU is light. However, more indepth research into the circuit dynamics and the optimal PI coefficients is required in future work.

The hardware circuitry is relatively simple. The voltage and sense circuit may seem complex at first glance; however, they are mostly composed of passive components. In any case, they are needed in the IPT system when $i_{\text {rec }}, u_{\text {rec }}$ or $Z_{\text {rec }}$ feedback is needed, even if phase synchronization is accomplished using other methods.

\section{Conclusion}

An impedance-based indirect phase synchronization method for the active rectifier in IPT systems is proposed. The operating principle of phase synchronization and the "tooth missing" phenomenon are discussed. Based simply on a PI controller that is executed at a low frequency, the proposed method directly controls the phase angle of the rectifier input impedance. When the impedance angle equals the target value, phase synchronization is automatically fulfilled. The PI coefficients are manually optimized and yield good static and dynamic performance. Advantages of the proposed method are discussed. 


\section{Acknowledgement}

This work was supported by Beijing Natural Science Foundation (No. 3212030).

\section{References}

1. M. Fu, C. Ma, X. Zhu: A Cascaded Boost-Buck Converter for High-Efficiency Wireless Power Transfer Systems. IEEE Transactions on Industrial Informatics, 10(3), 1972-1980 (2014).

2. M. Kato, T. Imura, Y. Hori: Study on maximize efficiency by secondary side control using DC-DC converter in wireless power transfer via magnetic resonant coupling. In: 2013 World Electric Vehicle Symposium and Exhibition (EVS27), pp. 1-5.

3. U. K. Madawala, D. J. Thrimawithana: A Bidirectional Inductive Power Interface for Electric Vehicles in V2G Systems. IEEE Transactions on Industrial Electronics, 58(10), 47894796 (2011).

4. D. J. Thrimawithana, U. K. Madawala, M. Neath: A Synchronization Technique for Bidirectional IPT Systems. IEEE Transactions on Industrial Electronics, 60(1), 301-309 (2013).

5. R. Mai, Y. Liu, Y. Li, P. Yue, G. Cao, Z. He: An Active-Rectifier-Based Maximum Efficiency Tracking Method Using an Additional Measurement Coil for Wireless Power Transfer. IEEE Transactions on Power Electronics, 33(1), 716-728 (2018).

6. E. Ozalevli et al.: A Cost-Effective Adaptive Rectifier for Low Power Loosely Coupled Wireless Power Transfer Systems. IEEE Transactions on Circuits and Systems I: Regular Papers, 65(7), 2318-2329 (2018).

7. Y. Liu, U. K. Madawala, R. Mai, Z. He: An Optimal Multivariable Control Strategy for Inductive Power Transfer Systems to Improve Efficiency. IEEE Transactions on Power Electronics, 35(9), 8998-9010 (2020).

8. S. Jia, C. Chen, P. Liu, S. Duan: A Digital Phase Synchronization Method for Bidirectional Inductive Power Transfer. IEEE Transactions on Industrial Electronics, 67(8), 6450-6460 (2020).

9. L. Cheng, W. Ki, Y. Lu, T. Yim: Adaptive On/Off Delay-Compensated Active Rectifiers for Wireless Power Transfer Systems. IEEE Journal of Solid-State Circuits, 51(3), 712-723 (2016).

10. Young-Jun Park et al.: A design of inductive coupling wireless power receiver with high efficiency Active Rectifier and multi feedback LDO regulator. In: 2016 IEEE Wireless Power Transfer Conference (WPTC), pp. 1-4.

11. Y. Jiang et al.: Phase-Locked Loop Combined With Chained Trigger Mode Used for Impedance Matching in Wireless High Power Transfer. IEEE Transactions on Power Electronics, 35 (4), 4272-4285 (2020).

12. G. Zhu, D. Gao: High-Frequency Voltage and Current Sense Circuits for Inductive Power Transfer Systems. IEEE Transactions on Power Electronics, 35(11), 11352-11362 (2020). 